\title{
Tratamiento de complicaciones post-cirugías antiincontinencia con "sling"
}

\author{
Palma P*, Riccetto C*, Miyaoka R*, Olivares JM**, Bigozzi MA***. \\ *Servicio de Urología de la Facultad de Medicina de la Universidad Estatal de Campinas- UNICAMP, Brasil.** \\ Servicio de Urología. ***Servicio de Tocoginecología. Policlínica Bancaria 9 de Julio. Buenos Aires Argentina.
}

Actas Urol Esp. 2008;32(7):737-744

\section{RESUMEN}

TRATAMIENTO DE COMPLICACIONES POST-CIRUGÍAS ANTIINCONTINENCIA CON "SLING".

Las complicaciones postoperatorias de la cirugía antiincontiencia, han comenzado a publicarse paralelamente al aumento proporcional del uso de la técnica de cabestrillo para la Incontinencia de orina de esfuerzo. En este trabajo de revisión describimos las complicaciones más frecuentes, como la obstrucción infravesical, el hematoma pélvico, lesiones uretrales y vesicales, con el fin de facilitar a quienes reproducen esta técnica una herramienta de diagnóstico y tratamiento para estas complicaciones.

Palabras clave: Cirugía antiincontinencia. Complicaciones. Mallas.

\section{ABSTRACT}

TREATMENT OF POST-SURGICAL COMPLICATIONS ANTIINCONTINENCE WITH "SLING"

Reports on postoperative complications of anti-incontinence surgery followed the widespread use of synthetic slings. In this paper we describe the more frequent complications, such as obstruction, pelvic hematoma, bladder and urethral injuries, to facilitate the management of these complications.

Keywords: Anti-incontinence surgery. Complications. Mesh.

$\mathrm{E}$ lempleo de los "cabestrillos" para el tratamiento de la Incontinencia Urinaria de Esfuerzo, fue propuesto en la primera mitad del siglo $\mathrm{XX}$, pero adquirió popularidad recientemente, a partir de la década del '70. Esto se relacionó directamente con el progreso del conocimiento en la fisiopatología de la Incontinencia Urinaria de Esfuerzo y, en especial, el estudio urodinámico. A pesar de la indicación clásica de los "cabestrillos", relacionada a los casos de insuficiencia esfinteriana intrínseca; su indicación en portadoras de hipermobilidad uretral, se torno cada vez más común, en virtud de los excelentes resultados de esta técnica después de un seguimiento prolongado, y más aún cuando se comparaba con las colposuspensiones abdominales, con las suspensiones endoscópicas del cuello vesical y con las colpoperineoplastías vaginales.

El aumento de la casuística, en tanto, determinó un crecimiento directamente proporcional de los reportes sobre la morbilidad de los "cabestrillos", donde se destacó las disfunciones vesicales y la obstrucción infravesical. Otro posible determinante de este hecho, es la gran variedad de materiales propuestos para la confección de los "cabestrillos", con un comportamiento biológico muy diverso.

\section{RESEÑA HISTÓRICA}

Históricamente se utilizaron diversos materiales, como tejidos corporales (fascia lata 34, fascia de rectos anteriores 35 ) con el objeto de reducir las erosiones, sin embargo, la disección de los tejidos aumentaba el tiempo quirúrgico y la morbilidad de la cirugía, por lo que fueron quedando en desuso paulatinamente.

Distintos materiales sintéticos no reabsorbibles se emplearon para el tratamiento de la incontinencia de orina de esfuerzo como Marlex, Mersilene, Teflón, Silastic, Prolene, Nylon, Gore - Tex. Los resultados obtenidos fueron disímiles, en función del material empleado, ya que algunos no eran bien 
tolerados y presentaban mayores tasas de infecciones y erosiones a nivel vaginal y uretrovesical.

Desde final de los años setenta hasta principios de los 90 los cabestrillos se restringían a las incontinencias recidivadas, ya que si bien mostraban tasa de curación del 91\%, se consideraban técnicas con alto riesgo de erosión y obstrucción miccional postoperatoria.

En 1995 Ulmsten propone una nueva técnica llamada "tension free vaginal tape" esta técnica se baso en que siendo un procedimiento sin tensión, disminuyó el riesgo de erosión del cabestrillo como así también las tasas de retenciones y dificultad urinaria en el posoperatorio.

Otro factor importante fue la elección de la uretra media o distal en lugar del cuello vesical para la colocación del cabestrillo, como la utilización de mallas de polipropileno monofilamento macroporo, siendo este, un material que genera tasas bajas de erosiones, infecciones y rechazos.

Dentro de las complicaciones de las dos técnicas quirúrgicas más utilizadas el cabestrillo transobturatriz (TOT) y el cabestrillo retropúbico de la porción media de la uretra (TVT), se observa en el TOT, una reducción significativa de las retenciones miccionales postoperatorias y de la incontinencia de urgencia de novo, esta retenciones parecen estar relacionadas con el tipo de cincha y la técnica postoperatoria.

En el estudio de Deng et al. (2007) sobre un total de 11.806 pacientes, donde se comparó las vías de abordaje TVT y TOT y sus complicaciones, se informó el 7.8 \% de complicaciones (928 pacientes), correspondían a 700 pacientes para la vía TVT, 66 SPARC, 1 TVT-O, 149 Obtape y 12 MONARC, demostrándose que la técnica transobturatriz presenta menos complicaciones postoperatorias.

\section{OBSTRUCCIÓN INFRA-VESICAL Fisiopatologia}

La obstrucción infravesical post "cirugía con cabestrillo", puede ocurrir por dos mecanismos básicos que, eventualmente, pueden coexistir en la misma paciente: (a) tensión exagerada aplicada sobre la uretra y (b) subvalorización de prolapsos urogenitales asociados en el preoperatório.

Entre el detrusor y la mucosa vesical, existe una capa independiente de músculo liso y elastina que se prolonga a partir del trígono, llamada anillo tri- gonal, que es considerado el principal determinante del cierre del cuello vesical durante el llenado ${ }^{10}$. De forma contraria, durante la micción, la contracción coordinada del anillo trigonal determina el afinamiento del cuello vesical, facilitando el vaciamiento. Este mecanismo es mediado básicamente por fibras alfa-adrenérgicas provenientes de centros de la médula tóraco-lumbar (T11 a L2) y conducidas a través del nervio hipogástrico ${ }^{32}$. Los "cabestrillos" pubovaginales clásicos, aplicados en la región del cuello vesical, dificultan este proceso, determinando, habitualmente, un aumento significativo de la presión del detrusor durante la micción. Así, pequeñas diferencias en la tensión aplicada sobre el "cabestrillos", pueden alterar este equilibrio de presiones, expresándose en forma de retención urinaria o, más frecuentemente, a través de síntomas irritativos urinarios ${ }^{15}$.

El soporte anatómico de la uretra, del cuello vesical y de la pared posterior de la vejiga, derivan primariamente de la fascia del músculo elevador del ano, que se inserta lateralmente en el arco tendíneo, junto a la rama ísquiopubiana. Alrededor de la uretra, condensaciones de esta fascia, originan los ligamentos uretropélvicos y pubouretrales ${ }^{25}$. Posteriormente, la fascia del músculo elevador del ano se extiende sobre la pared vesical posterior hasta el cervix uterino, recibiendo la denominación de fascia pubocervical, responsable del soporte de estas estructuras, también como de la pared vaginal anterior (Fig. 1). El mantenimiento del eje uretro-vesical, determinado por la fascia pubocervical, es fundamental para la micción normal. Cuando existe alteración de la sustentación vesical, sea por laceración central de la fascia pubocervical o por defecto de su inserción lateral en el arco tendíneo, la confección aislada de un "cabestrillo", con una tensión adecuada, puede intensificar la rotación vesical durante el aumento de la presión abdominal en torno a la uretra fija (Fig. 2), determinando la obstrucción infravesical $^{23}$. La incidencia de obstrucción esta, directamente, relacionada con el grado de prolapso, estimándose una incidencia de hasta $72 \%$ en los casos de cistocele grado 3 y $4^{2}$. Así, pacientes con clínica de obstrucción infra-vesical post "cirugía con cabestrillo", deben ser cuidadosamente evaluadas en cuanto a la presencia de distopías genitales, debido a que pueden estar directamente relacionadas a este cuadro clínico. 


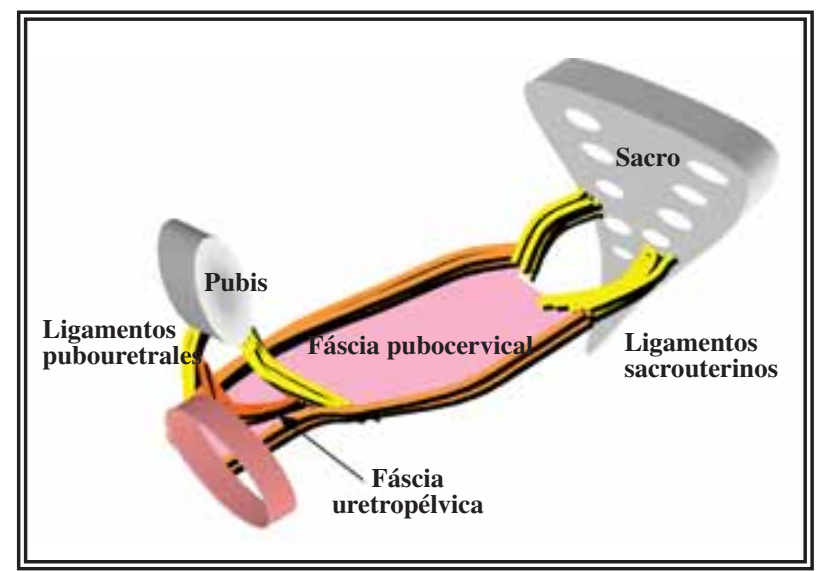

FIGURA 1. El soporte vesical, promovido por la fascia pubocervical, debe ser cuidadosamente evaluado antes de la realización del "cabestrillo", a fin de evitar la obstrucción infravesical.

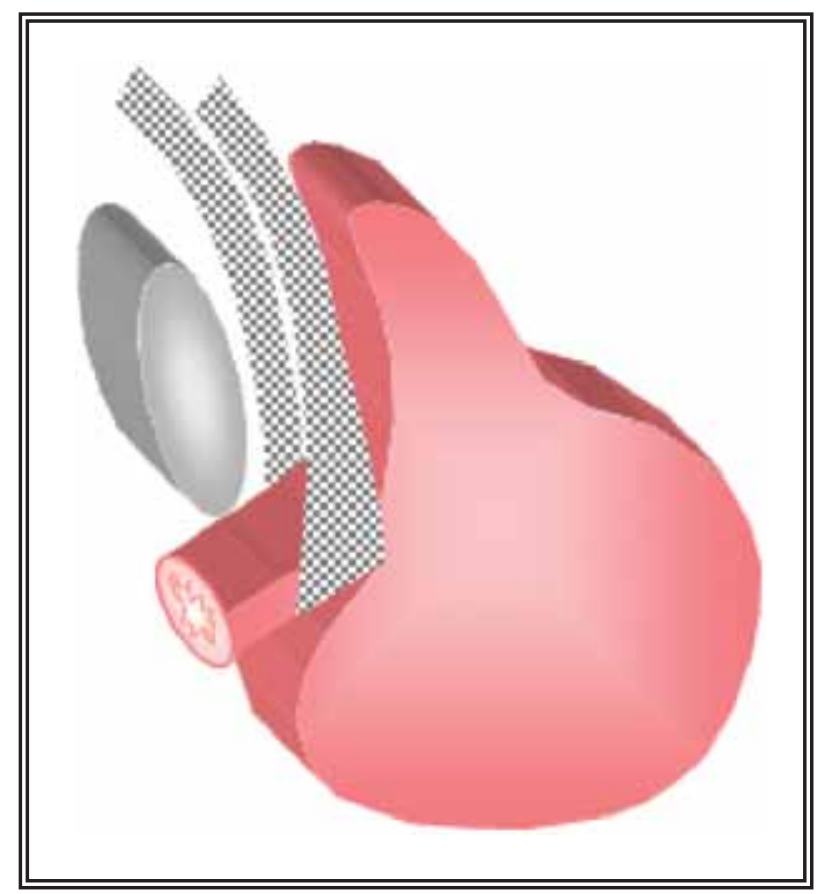

FIGURA 2. Obstrucción infravesical post "cirugia con cabestrillo", resultando cistocele moderado no corregido simultáneamente.

En cuanto a las situaciones descriptas no existe estenosis verdadera, raramente, el proceso obstructivo puede resultar de estrechamiento de la luz uretral, secundaria a suturas aplicadas próximas a la uretra, pérdida urinaria local o traumatismo por el catéter uretral. La estenosis es particularmente más frecuente con "cabestrillos" biológicos heterólogos y sintéticos, debido a la mayor incidencia de complicaciones infecciosas y de erosión uretral ${ }^{14-24}$.

\section{Diagnóstico}

Diversos estudios han demostrado un aumento significativo del diagnóstico de obstrucción infravesical en la mujer, sin embargo los criterios diagnósticos no se encuentran aún completamente esclarecidos. Los síntomas clásicos de chorro fino e intermitente, dificultad para iniciar la micción, son referidos por las mujeres en apenas $40 \%$ de los casos $^{17}$. De forma contraria, los síntomas irritativos, incluido disuria, polaquiuria, urgencia e incontinencia de urgencia son referidos por el $75 \%$ de las pacientes con esta condición ${ }^{7}$. En la anamnesis se debe investigar, la necesidad de la paciente de adquirir determinado decúbito o reducir digitalmente su prolapso genital para facilitar el vaciamiento vesical ${ }^{12}$.

En el examen físico general se debe verificar la presencia de globo vesical palpable. El examen ginecológico debe ser realizado cuidadosamente e incluir la palapación bimanual y el examen especular. Se debe verificar la presencia de cistocele, si es necesario con la paciente en posición semi-ortostática. En los casos donde hubo hipercorrección, es común observar angulación uretral exagerada en relación al cuerpo vesical y, en algunos casos, retracción del meato uretral para el interior de la vagina.

Como la mayoría de las pacientes no tienen estenosis verdadera, el empleo de la uretroscopía para su diagnóstico no es necesario y, generalmente, no es recomendado. A pesar que la uretrocistografía miccional, en posición ortostática, pueda documentar el punto de obstrucción, se trata de un examen estático, que frecuentemente disminuye mucho su sensibilidad en relación a la fluoroscopía.

No existe, hasta el momento, consenso en cuanto a los parámetros urodinámicos que deban ser utilizados para establecer este diagnóstico ${ }^{3}$. Varios autores propusieron la definición de la obstrucción con base en criterios exclusivamente urodinámicos (estudio flujo-presión), en general correlacionando el flujo urinario máximo y la presión del detrusor en flujo máximo ${ }^{11}$. En tanto, debido a la gran amplitud del patrón miccional femenino considerado normal, establecer parámetros urodinámicos que puedan caracterizar el diagnóstico de obstrucción infra-vesical en mujeres, no es simple ${ }^{4}$. A pesar de estas dificultades fue propuesto, recientemente, por Blaivas \& Groutz, un nomograma para la evaluación de las pacientes con sospecha de obstrucción infravesical (Fig. 3), empleando como parámetros, la presión del 


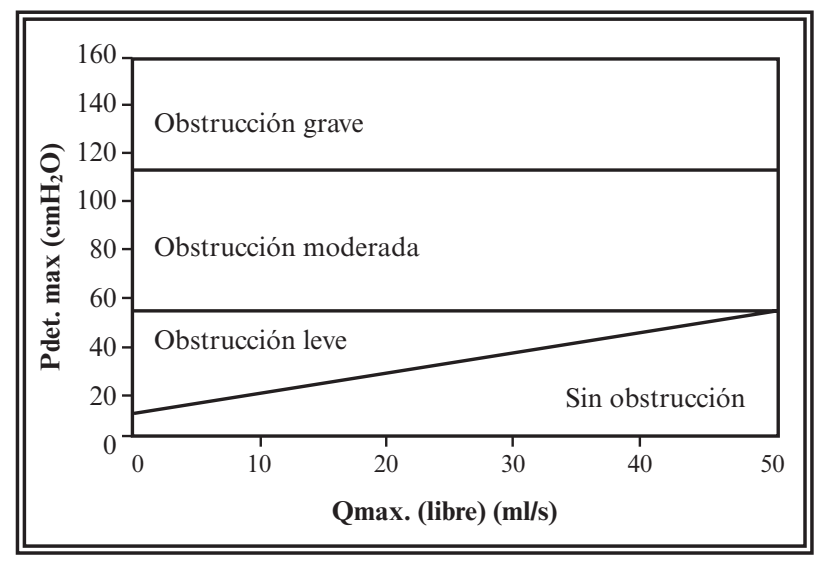

FIGURA 3. Nomograma de Blaivas \& Groutz para el diagnóstico de obstrucción infravesical en la mujer.

detrusor máxima alcanzada durante la micción y el flujo máximo obtenido previamente al cateterismo uretral. A pesar del rigor estadístico y de la coherencia con la fisiopatología, su valor aún se encuentra indeterminado ${ }^{3}$. El empleo de la videourodinamia, permite asociar los parámetros urodinámicos con la fluoroscopía, parece representar, hasta el momento, el método de mayor sensibilidad para el diagnóstico, además de permitir la demostración del punto exacto de la obstrucción. La videourodinamia posibilita, también, la evaluación precisa de la influencia de los prolapsos urogenitales sobre el proceso, a través de la simulación de la micción después de su corrección, por medio de la introducción de un pesário vaginal antes de la fase miccional ${ }^{9-18}$.

\section{Tratamiento}

Como todavía no existe consenso acerca del diagnóstico, a pesar de las tentativas de normatización basadas en criterios urodinámicos objetivos, cualquier análisis crítico de los resultados obtenidos, con diferentes formas de tratamiento, la obstrucción infra-vesical en la mujer presenta limitaciones. El abordaje terapéutico será distinto, dependiendo del tiempo entre el "cabestrillo" y la sospecha de obstrucción.

La aparición de disfunción miccional o de retención urinaria temporaria después de los "cabestrillos" pubovaginales es relativamente frecuente ${ }^{16-29}$. La conducta inicial debe ser conservadora hasta los dos primeros meses después del procedimiento. Después de la desaparición del dolor y de la disminución del edema local, la mayoría de las veces ocurre la resolución espontánea de estos síntomas, sin la necesidad de intervención ${ }^{28}$. La retención urinaria importante debe ser conducida con auto-cateterismo intermitente durante este período. Aquellos casos en que la sintomatología persiste deben ser evaluados criteriosamente. El diagnóstico de inestabilidad vesical, no es significativo, desde el punto de vista terapéutico, debido a que estas mujeres no responden al uso de anti-colinérgicos $\mathrm{u}$ otras conductas conservadoras ${ }^{16}$. El tratamiento clásico de la obstrucción infra-vesical post "cirugía con cabestrillo" es la uretrolisis. Varias técnicas de uretrolisis fueron propuestas, con acceso abdominal o vaginal (suprameatal o transvaginal). Independientemente de la vía de abordaje, este procedimiento tiene por objetivo disecar todas las eventuales adherencias periuretrales, particularmente, aquellas existentes entre la uretra y la cara inferior de la sínfisis púbica (Fig. 4) ${ }^{33}$. Los índices de éxito de las diversas técnicas de uretrolisis son semejantes, y varían entre 70 a $80 \%{ }^{5-19,20-22}$. La uretrolisis transvaginal ofrece como ventaja menor morbilidad en relación a la retropúbica $^{19}$, sin los riesgos de sangrado involucrados en el acceso suprameatal, en virtud del plexo venoso del clítoris. El éxito de la uretrolisis transvaginal parece mayor en la obstrucción secundaria a la suspensión con aguja (71\%) y uretropéxia retropúbica (63\%), cuando se compara aquella secundaria al "sling" pubo-vaginal $(50 \%)^{19}$. En casos con fibrosis periuretral importante o despúes del fracaso de uretrolisis anterior, es indicada la interposición de un segmento fibroadiposo del labio mayor

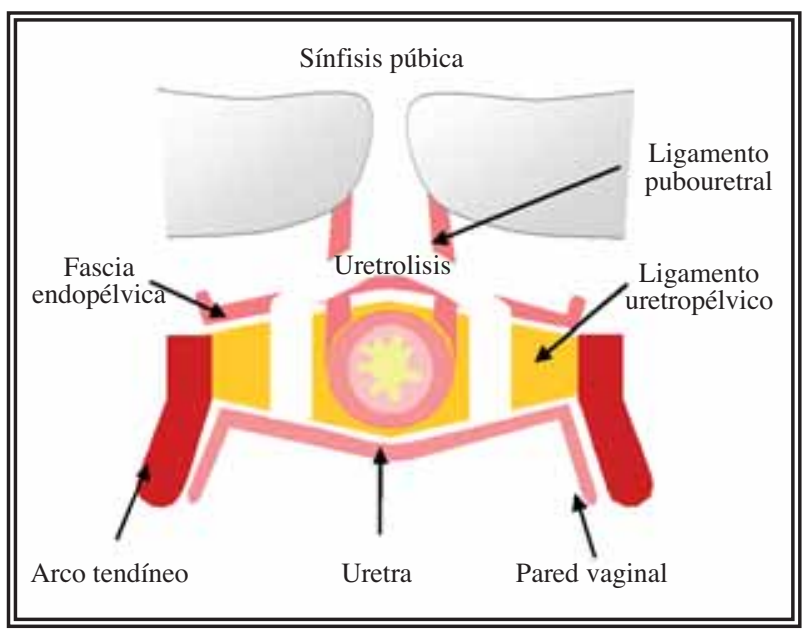

Figura 4. En la uretrolisis transvaginal, se realiza una disección de las adherencias existentes entre la uretra y/o el pubis. 
(Martius), pediculado en la arteria pudenda externa entre la uretra y la sínfisis púbica (Fig. 5a y 5b). Esta maniobra representa un importante recurso a fin de evitar que el proceso cicatrizal cause nuevas adherencias de la uretra a la cara inferior de la sínfisis púbica y eventualmente puede ser propuesto en el primer abordaje de la paciente ${ }^{20}$. A pesar, que algunos autores, indiquen de rutina la asociación de un nuevo "cabestrillo" asociado a uretrolisis, la posibilidad de incontinencia después de este procedimiento es infrecuente, en menos de $5 \%$ de los $\operatorname{casos}^{30}$.

Eventualmente, la posibilidad de cateterismo intermitente limpio definitivo, debe ser considerada, como una opción terapéutica más adecuada en pacientes seleccionadas, como en aquellas portadoras de hipocontractilidad vesical importante, posterior a repetidas fallas en uretrolisis anteriores o en pacientes con comorbilidades importantes que imposibiliten cualquier procedimiento quirúrgico.
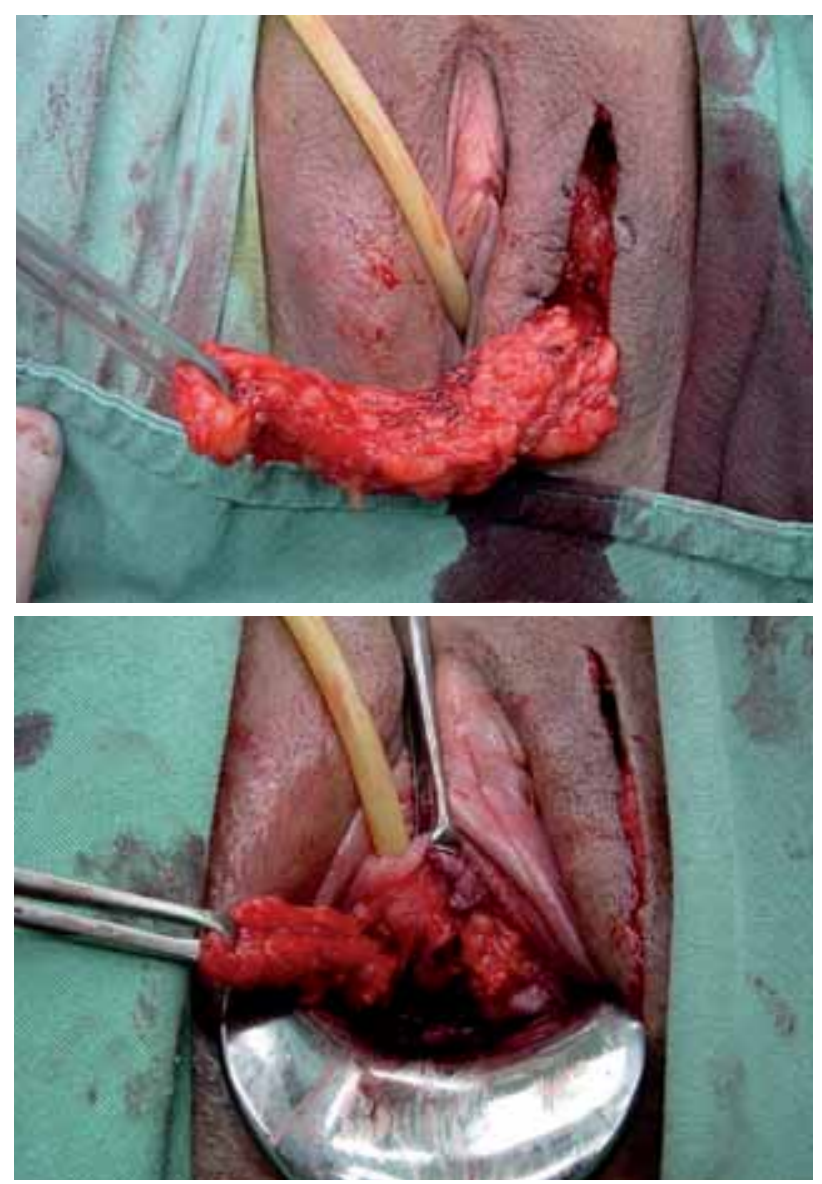

FIGURA 5 A Y B: El colgajo fibroadiposo de Martius puede ser interpuesto entre la uretra y la sinfisis pubica, con la finalidad de evitar la recidiva de la obstrucción posturetrolisis transvaginal.

\section{HEMATOMA PÉLVICO}

Cualquier procedimiento quirúrgico puede estar acompañado de sangrado importante ocasionado por condiciones particulares de la paciente, como uso de anti-agregantes plaquetários o por peculiaridades del propio acto quirúrgico. En la cirugía antiincontinencia con colocación de un "cabestrillo", el momento más sujeto a sangrado es durante la perforación de la fascia endopélvica por la aguja que irá a suspender los extremos del "cabestrillo". Entre tanto, se debe estar preparado para controlar el sangrado intra-operatorio en todos los momentos.

Una técnica quirúrgica meticulosa y el conocimiento de la anatomía son principios fundamentales para evitar una hemorragia importante.

En algunas situaciones, como en la presencia de sangrado arterial o venoso importante, se torna necesaria una hemostasia más vigorosa, caso contrario, medidas más conservadoras son suficientes. Katske y Raz describieron la introducción de un catéter Foley intravaginal e insuflaron el balón con 30 a $90 \mathrm{ml}$. de agua, con el fin de controlar hemorragias importantes (Fig. 6) ${ }^{13}$. En otras situaciones, como sangrado del espacio retropúbico, se puede optar por la introducción de un tapón de gasas temporalmente en este lugar, que frecuentemente provee un taponamiento efectivo. Suturas simples son necesarias en presencia de puntos sangrantes, de difícil coagulación, en la pared vaginal y uretral.

Sangrados importantes, con formación de hematomas capaces de desviar estructuras pélvicas, pueden ocurrir muy raramente. En estos casos, el drenaje de los mismos, en general es a través del acceso transvaginal (Fig. 7 a y b).

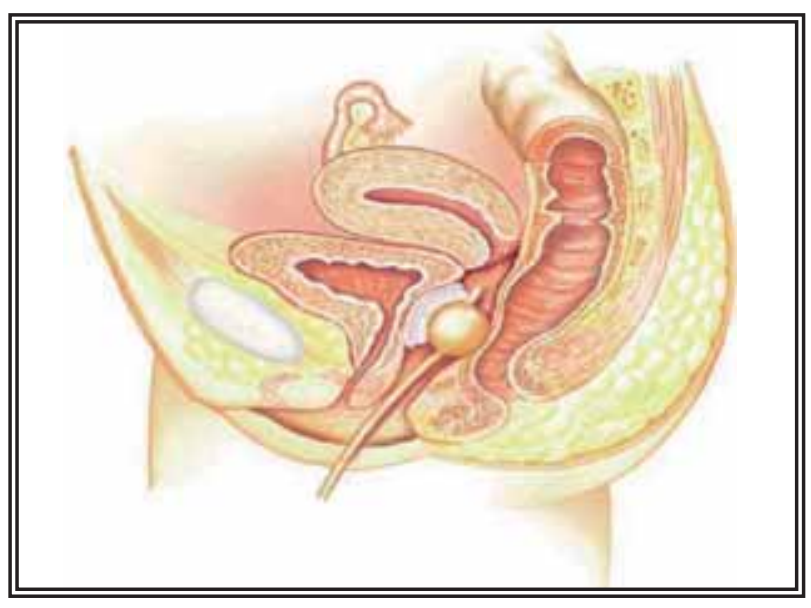

FIGURA 6. Catéter foley intravaginal a fin de taponar el sangrado. 

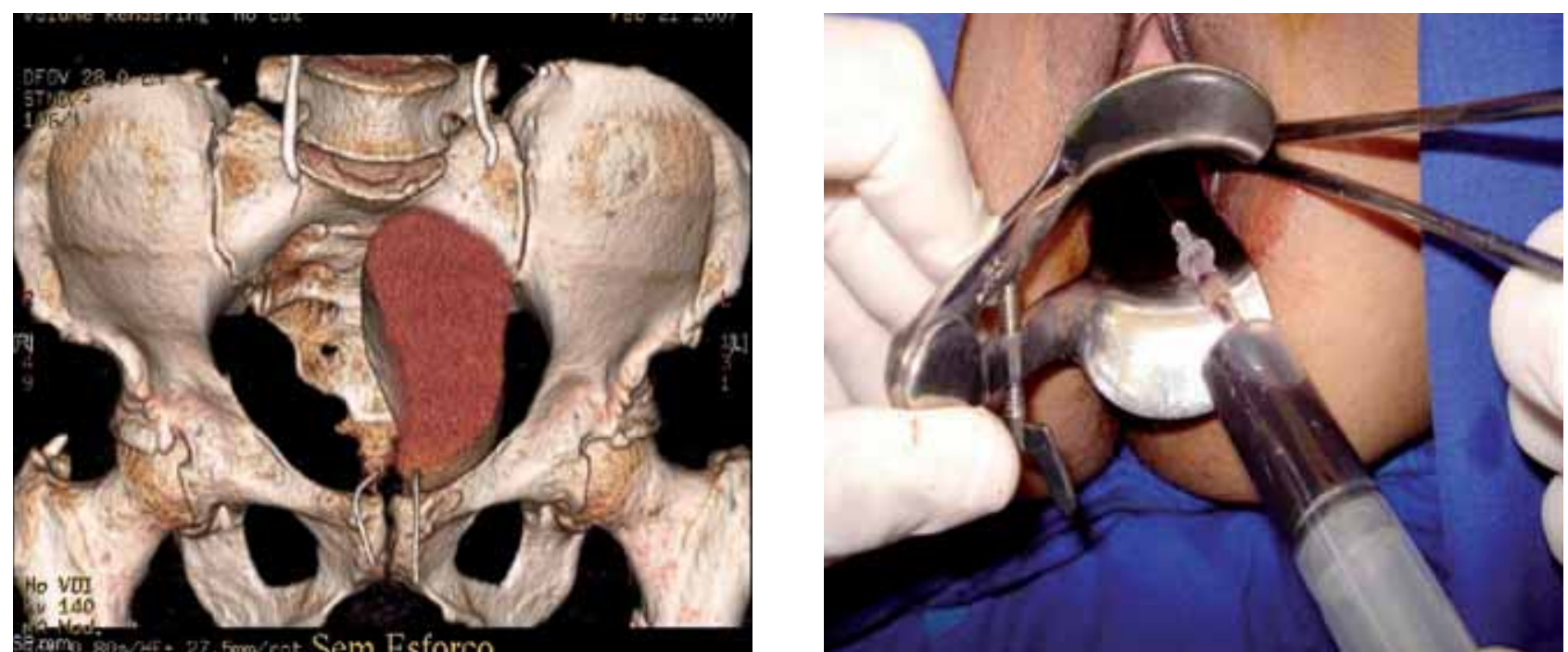

FIGURA 7 A Y B. a) Reconstrucción 3D de hematoma pélvico que despalza vejiga post cirugía de cabestrillo. b) Drenaje de hematoma por via transvaginal

\section{EROSION}

Diversos materiales sintéticos han sido utilizados para la confección de los "cabestrillos". El objetivo del empleo de estos materiales es disminuir la utilización del material autólogo, diminuyendo así el dolor en el post operatorio, y el uso de la fascia cadavérica, teóricamente, diminuyendo la transmisión de infecciones.

Complicaciones post-operatorias como infección y erosión ocurren más comúnmente cuando se utilizan materiales sintéticos (Fig. 8) y, normalmente, se asocian a dificultades en la incorporación del material en el tejido subepitelial. La literatura reporta índices de erosión de hasta $23 \%$ para los "cabestrillos" sintéticos, en períodos post operatorios de hasta 2 años. Recientemente, fue descripto altos índices de infección y erosión uretral con la utilización del pericardio bovino.

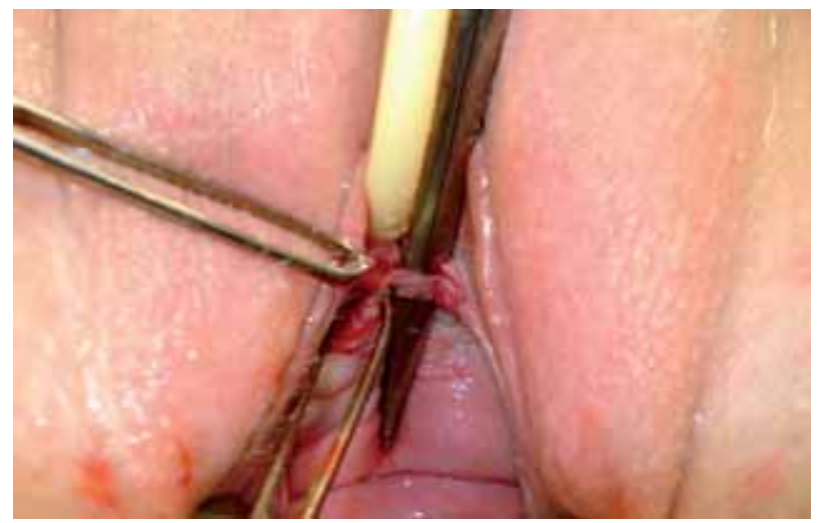

FIGURA 8. Se observa erosión del "cabestrillo" de pericardio bovino, a través de la pared vaginal.
La etiología exacta de estas erosiones no es conocida. Algunas teorías sugieren una mala calidad y vascularización deficiente de los tejidos que envuelven el material implantado, desencadenando infección post operatoria y demasiada tensión en los puntos de suturas en la pared vaginal.

Las pacientes con erosión vaginal o uretral del "cabestrillo", frecuentemente se presentan con quejas de dispareunia, secreción sanguínea uretral y urgencia miccional. Cuando la erosión ocurre a nivel de la vejiga, las quejas más relatadas son síntomas urinarios irritativos e infecciosos. Entre tanto, se puede diagnosticar, erróneamente, como instabilidad u obstrucción post operatoria. En los casos donde hay exposición del material por la pared vaginal o uretral, normalmente son identificados durante el examen físico cuidadoso. Las lesiones vesicales presentan un examen pélvico normal, lo que puede llevar a un retardo en el diagnóstico. Chai y Sklar demostraron el uso del cistoscópio flexíble para la realización de vaginoscopía, como método auxiliar en el diagnóstico de pequeñas erosiones. La uretrocistoscopía está indicada como método diagnóstico en las sospechas de erosiones uretrales y vesicales ${ }^{6}$.

La erosión post "cirugía con cabestrillo" pubovaginal típicamente resulta en síntomas de larga evolución antes del diagnóstico correcto. Clemens et al. sugieren que, pacientes que presentan dolor pélvico inespecífico, secreción vaginal y síntomas urinarios irritativos sin causa aparente y con historia de ciru- 
gía de tipo "cabestrillo", deben ser investigadas a través de un examen físico cuidadoso y uretrocistoscopía ${ }^{8}$.

El tratamiento de esta complicación, una vez diagnosticada, debe ser inmediato, con remosión del material sintético, inclusive suturas. En erosiones uretrales, se debe intentar identificar la mucosa uretral, realizar la reconstrucción con hilos absorbibles y mantener cateterismo uretral por un período no inferior a 7 días. Los riesgos que involucran la remosión del "cabestrillo", son la lesión vesical, hemorragia y recidiva de la incontinencia. Bent et al. Reportaron 26 \% de incontinencia en estas pacientes $^{1}$. Contradictoriamente, Summit et al. No detectaron ningún caso de recidiva en el grupo donde el material fue removido ${ }^{26}$. Clemens et al. Destacan que pacientes con erosión vaginal y uretral, presentan posibilidades elevadas de recidiva de incontinencia urinaria ${ }^{8}$. Estos autores sugieren un procedimiento anti-incontinencia, en el mismo momento de la retirada del "cabestrillo", en aquellos casos donde la reacción inflamatoria local no es intensa y la paciente refiere perdidas.

Timmons e Addison reportaron tratamiento conservador de 16 pacientes que presentaron erosión del "cabestrillo" sintético (Mersilene). El abordaje quirúrgico involucró la extracción del fragmento erosionado y la cobertura del área expuesta con un segmento da pared vaginal. Estos autores verificaron un índice de éxito alrededor de $56 \%{ }^{27}$. Un abordaje semejante fue descripto por Myers et al. Que obtuvieron éxito en $75 \%$ de los pacientes $^{21}$. El tratamiento conservador puede ser adoptado, entre tanto, es necesario que no exista infección local. Se debe tener especial cuidado con la introducción de antibioticoterápia y lavajes abundantes de la pared vaginal, a fin de reducir al máximo la concentración bacteriana, además de hemostasia meticulosa y realización de suturas sin tensión y con puntos separados.

En la presencia de erosión intravesical, Clemens et al., realizaron la extracción del cabestrillo por vía uretral, seccionándolo en el interior de la vejiga, para luego exteriorizarlo ${ }^{8}$.

Con el fin de disminuir al máximo los episodios de infección y erosión en los "cabestrillos" sintéticos, optamos por realizar antisepsia cuidadosa del canal vaginal en el preoperatorio inmediato y en la sala de cirugía, introducción del material sintético en solución antibiótica durante 15 minutos y utilización de segmentos de pared vaginal bien vascularizados para la cobertura del "cabestrillo" implantado.

\section{Algoritmo diagnóstico y terapéutico de las erosiones del tracto genito-urinario (modificado de Clemens JQ et al, Urology, 2000) ${ }^{8}$}

\section{PRESENTACIÓN CLÍNICA}

- Dolor a la palpación de la vagina y/o de la uretra

- Secreción y sangrado vaginal

- Infección urinaria a repetición

- Síntomas miccionales irritativos

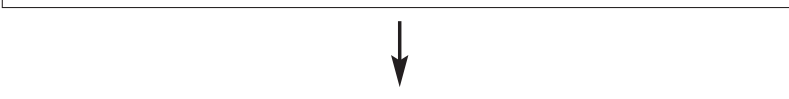

\section{EVALUACIÓN}

Examen pélvico: investigar la presencia de dolor a la palpación de la pared vaginal anterior, presencia de material extraño en la pared

- Cistoscopía: evaluar la presencia de reacción inflamatoria y de cuerpo extraño

- Vaginoscopía: auxiliar en el diagnóstico de erosiones vaginales pequeñas

\begin{tabular}{|ccc|}
\hline & \multicolumn{1}{c|}{ TRATAMIENTO } & \\
\hline & EROSIÓN & EROSIÓN \\
EROSIÓN & VAGINAL & URETRAL \\
VESICAL & Abordaje vaginal & - Abordaje vaginal \\
- Remoción del & - & \\
material intra- & - Remoción de & - Remoción de \\
vesical a través & todo material & todo material \\
de la vía uretral & sintético & sintético \\
& - Cateter uretral & - Si es posible \\
& por 1-2 dias & cerrar la uretra, \\
& & utilizando hilos \\
& & absorbibles \\
& & Catéter uretral \\
& & debe permane- \\
& & cer por lo \\
& & menos durante \\
& & 7 días \\
& &
\end{tabular}

\section{LESIONES VESICALES Y URETRALES Vejiga}

Lesiones vesicales durante la cirugía anti-incontinencia ocurren más frecuentemente en la base de la vejiga, en cambio, las lesiones clásicas del pasaje de la aguja de suspensión supra-púbica, son las perforaciones antero-laterales. 
El cuidado de vaciar la vejiga inmediatamente antes del pasaje de la aguja puede reducir las probabilidades de lesión del órgano. La utilización de un recipiente colector de orina conectado a una sonda de Foley puede demostrar la presencia de hematuria, sugiriendo lesión vesical. La cistoscopía operatoria determinará el lugar y la extensión de la lesión.

Donde se observe suturas intravesicales, debido al pasaje inadvertido de la aguja de suspensión, estas deben ser removidas inmediatamente, dejando sonda vesical por un periodo de 7 dias. Lesiones vesicales no identificadas en el intraoperatorio pueden resultar en inestabilidad del detrusor post operatoria, infección del tracto urinario a repetición o formación de cálculos.

Lesiones más extensas deben ser tratadas a través de suturas de la pared vesical en dos planos, utilizando hilos absorbibles.

\section{Uretra}

Las lesiones uretrales deben ser reparadas en el mismo acto quirúrgico, a fin de evitar secuelas postoperatorias como la formación de divertículos o fístulas $^{31}$. En lesiones superficiales, sin abertura de la mucosa uretral, se puede optar por sutura simple, en plano único, con hilo absorbible. En la presencia de lesiones más complejas, se opta por sutura en dos planos y aproximación de la fascia periuretral, pudiéndose utilizar un colgajo de Martius en la confección de neouretras.

\section{REFERENCIAS}

1. Bent AE, Ostergard DR, Zwich-Zaffuto MT. Tissue reaction to expanded polytetrafluoroethylene suburethral sling for urinary incontinence: clinical and histologic study. Am J Obstet Gynecol. 1993;169(5):1198-1204.

2. Romanzi LJ, Chaikin DC, Blaivas JG. The effect of genital prolapse on voiding. J Urol. 1999;161(2):581-586.

3. Blaivas JG, Groutz A. Bladder Outlet obstruction nomogram for women with lower urinary tract symptomatology. Neurourol Urodyn 2000;19(5):554-564.

4. Chaikin DC, Groutz A, Blaivas JG. Predicting the need for anti-incontinence surgery in continent women undergoing repair of severe urogenital prolapse. J Urol. 2000;163(2):531-534.

5. Carr LK, Webster GD. Bladder outlet obstruction in women. Urol Clin North Am. 1996;23(3):385-391.

6. Chai TC, Sklar GN. Use of the flexible cystoscope as a vaginoscope to aid in the diagnosis of artificial sling erosion. Urology. 1999;53(3):617-618.

7. Chassagne S, Bernier PA, Haab F, Roehborn CG, Reisch SJ, Zimmern PE. Proposed cutoff values to define bladder outlet obstruction in women. Urology. 1998;51(3):408-411.

8. Clemens JQ, DeLancey JO, Faerber GJ, Westney OL, Mcguire EJ. Urinary tract erosions after synthetic pubovaginal slings: diagnosis and management strategy. Urology. 2000;56(4):589-595.

9. Coates KW, Harris RL, Cundiff GW, Bump RC. Uroflowmetry in women with urinary incontinence and pelvic organ prolapse. Brit J Urol. 1997;80(2):217-221.

10. DeLancey JO. Anatomy and physiology of urinary continence. Clin Obst Gynec. 1990;33(2):298-302
11. Farrar DJ, Osborne JL, Stephenson TP, Whiteside CG, Weir J, Berry J, et al. A urodynamic view of bladder outflow obstruction in the female: factors influencing the results of treatment. Br J Urol. 1975:47(7):815-825.

12. Nitti VW, Tu LM, Gitlin J. Diagnosing bladder outlet obstruction in women. J Urol. 1999;161(5):1535-1540.

13. Katske FA, Raz S. Use of Foley catheter to obtain transvaginal tamponade. Urol Urotech. 1987; May 18.

14. Leach GE, Dmochowski RR, Appell RA, Blaivas JG, Hadley HR, Luber KM, et al. Female stress urinary incontinence clinical guidelines panel summary report on surgical management of female stress urinary incontinence. The American Urological Association. J Urol. 1997;158(3 Pt1):875-880.

15. Lemack GE, Zimmern P. Pressure Flow analysis may aid in identifying women with outflow obstruction. J Urol. 2000;163(6):1823-1828.

16. Austin P, Spyropoulos E, Lotenfoe R, Helal M, Hoffman M, Lockhart JL. Urethral obstruction after anti-incontinence surgery in women: evaluation, methodology and surgical results. Urology. 1996;47(6):890-894.

17. Massey JA, Abrams PH. Obstructed voinding in the female. Brit J Urol. 1988; 61(1):36-42.

18. Mattox TF, Bhatia NN. Urodynamic effects of reducing devices in women with genital prolapse. Int J Urogynec 1994;5:283-45.

19. Foster HE, McGuire EJ. Management of urethral obstruction with transvaginal urethrolysis. J Urol. 1993;150(5 Pt1):1448-1451.

20. Cross CA, Cespedes RD, English SF, McGuire EJ. Transvaginal urethrolysis for urethral obstruction after anti-incontinence surgery. J Urol. 1998;159(4):11991201.

21. Myers DL, LaSala CA. Conservative surgical management of Mersilene mesh suburethral sling erosion. Am J Obstet Gynecol. 1998;179(6):1424-1429.

22. Nitti V, Raz S. Obstruction following anti-incontinence procedure: diagnosis and treatment with transvaginal urethrolysis. J Urol. 1994;152:93-98.

23. Nitti V. Bladder outlet obstruction in women. In Nitti V: Practical Urodynamics. New York, Saunders, 1998; p.197-210.

24. Palma PCR, Ikari O, D'Ancona CAL, Netto NRJr. Alça pubovaginal sintética no tratamento da IUE. J Bras Urol 1992; 18: 201-4.

25. Stothers L, Chopra A, Raz S. Vaginal reconstrutive surgery for female incontinence and anterior vaginal-wall prolapse. Urol Clin North Am 1995; 22: 641-55.

26. Summit RL, Bent AE, Ostergard DR, Harris TA. Suburethral sling procedure for genuine stress incontinence and low urethral closure pressure: a continued experience. Int Urogynecol. J 1992; 3:18-21.

27. Timmons CM, Addison WA. Mesh erosion after abdominal sacral colpopexy. J Pelvic Surg 1997; 3:75-80.

28. Carr LK, Webster GD. Voiding disfunction following incontinence surgery: diagnosis and treatment with retropubic or vaginal urethrolysis. J Urol. 1997;157: 821-823.

29. Weinberger MW, Ostergard DR. Long-term clinical and urodynamic evaluation of the polytetrafluoroethylene suburethral sling for treatment of genuine stress incontinence. Obst Gynec. 1995; 86(1):92-96.

30. Zaragoza MR. Expanded indications for the pubovaginal sling: treatment of type 2 or 3 stress incontinence. J Urol. 1996;156(5):1620-1631

31. Zimmern P, Schmidbauer CP, Leach GE, Staskin DR, Hadley HR, Raz S. Vesicovaginal and urethrovaginal fistulae. Semin Urol. 1986;4(1):24-29.

32. Zinner NR, Sterling AM, Ritter RC. Role of inner urethral softness in urinary continence. Urology 1980;16(1):115-117.

33. Video Clip de uretrolisis com asa de Collins. www.savefile.com/ files/1407058

34. Aldridge AH. Transplatation of fascia for relief of urinary stress incontinence. Am J Obstet Gynecol. 1942; 44:398-405.

35. Mcguire EJ, Lytton B. Pubovaginal sling procedure for stress incontinence. J Urol.1978;119(1):82-84.

36. Deng DY, Rutman M, Raz S, Rodriguez LV. Presentation and Management of Major Complications of Midurethral Slings: Are Complications Under-reported? Neurourol Urodyn 2007;26(1): 46-52

Correspondencia autor: Dr. P. Palma

Servicio de Urología. Facultad de medicina Universidad Estatal de Campinas-UNICAMP. Brasil.

Rua José Pugliesi Filho, 265 - Campinas,

Sao Paulo Brasil 13085-415

E-mail autor: ppalma@uol.com.br

Información artículo: Original - Urología femenina - Incontinencia

Trabajo recibido: marzo 2008

Trabajo aceptado: abril 2008 